\title{
A novel herbal treatment reduces depressive- like behaviors and increases brain-derived neurotrophic factor levels in the brain of type 2 diabetic rats
}

\author{
This article was published in the following Dove Press journal: \\ Neuropsychiatric Disease and Treatment \\ 29 November 2016 \\ Number of times this article has been viewed
}

\section{Chun Luo',* \\ Yuting Ke ${ }^{1, *}$ \\ Yanyan Yuan' \\ Ming Zhao' \\ Fuyan Wang' \\ Yisheng Zhang ${ }^{2}$ \\ Shizhong Bu'}

'Runliang Diabetes Laboratory, Diabetes Research Center, Ningbo University, ${ }^{2}$ Department of Gynaecology and Obstetrics, Ningbo Medical Center, Li Huili Eastern Hospital, Ningbo, Zhejiang. People's Republic of China

*These authors contributed equally to this work
Correspondence: Shizhong Bu Runliang Diabetes Laboratory, Diabetes Research Center, Ningbo University, 8I8 Fenghua Road, Ningbo 3152II, Zhejiang, People's Republic of China

Tel +8657487609607

Email bushizhong@nbu.edu.cn
Background: Radix Puerariae and hawthorn fruit have been demonstrated to treat diabetes. They offer potential benefits for preventing depression in diabetes.

Objective: The aim of this study was to investigate whether the combination of Radix Puerariae and hawthorn fruit (CRPHF) could prevent depression in a diabetic rat model generated by feeding the rats with a high-fat diet and a low-dose streptozotocin (STZ).

Methods: The CRPHF was provided by the Shanghai Chinese Traditional Medical University. Twenty-four rats were randomly divided into four groups: normal control, normal-givenCRPHF (NC), diabetic control, and diabetic-given-CRPHF (DC) groups. The type 2 diabetic model was created by feeding the rats with a high-fat diet for 4 weeks followed by injection of $25 \mathrm{mg} / \mathrm{kg} \mathrm{STZ}$. CRPHF was given at $2 \mathrm{~g} / \mathrm{kg} / \mathrm{d}$ to the rats of NC and DC groups by intragastric gavage daily for 4 weeks after the type 2 diabetic model was successfully created. Body weight, random blood glucose (RBG), oral glucose tolerance test, total cholesterol (TC), triglyceride (TG), high-density lipoprotein cholesterol (HDL-C), and low-density lipoprotein cholesterol (LDL-C) were measured during the study. Depressive-like behavior was evaluated at the end of the treatment by using the open field test (OFT), the elevated plus-maze test (EPMT), locomotor activity test (LAT), and forced swimming test (FST). Levels of extracellular signal-regulated protein kinase (ERK) and brain-derived neurotrophic factor (BDNF) in the prefrontal cortex were evaluated by using Western blot.

Results: 1) CRPHF reduced RBG and improved glucose tolerance in diabetic rats; 2) CRPHF reduced TC and TG but did not significantly change HDL-C or LDL-C in diabetic rats; 3) CRPHF reversed the loss in body weights observed in diabetic rats; 4) CRPHF reduced depressive-like behavior as measured by OFT, EPMT, LAT, and FST; 5) BDNF was upregulated, and ERK was activated in the prefrontal cortex of diabetic rats treated with CRPHF.

Conclusion: CRPHF has the potential of preventing depression in patients with diabetes.

Keywords: Puerarin, Crataegus, diabetes mellitus, depression, BDNF

\section{Introduction}

Diabetes mellitus (DM) and depression are both chronic and potentially life-threatening illnesses with a high prevalence in the modern era. The International Diabetes Federation reported that 382 million people suffered from DM worldwide in 2013, and this number would exceed 592 million in $<25$ years (Federation 2013). Similarly, in 2014, the World Health Organization estimated that depression affects $>350$ million people globally. Previous studies demonstrated that there is a bidirectional association 
between depression and type 2 diabetes. Depression is a major risk factor in the development of type 2 diabetes, ${ }^{1,2}$ and patients with diabetes show about twice the prevalence of depression compared with the general population. ${ }^{3}$ It is reported that the presence of diabetic complications, low monthly family income, negative life event, and poor social support were the statistically significant risk factors associated with depression in diabetic patients. ${ }^{4,5}$ However, the pathophysiological mechanism remains unclear. Further research is required to identify the physiological and pathological relations between diabetes and depression.

As depression is a common comorbidity characterized by suicidal tendency and recurrence of morbidity among patients with type 2 diabetes, there is a requirement to find effective drugs to prevent the onset of depression among people with type 2 diabetes. Previous studies have shown the therapeutic effects of some Traditional Chinese Medicine in the treatment of depression. ${ }^{6,7}$ The antidepressant effect of the ethanol extract of Radix Puerariae has been reported in mice exposed to cerebral ischemia reperfusion ${ }^{6}$ and in $\mathrm{C} 57 \mathrm{BL} / 6 \mathrm{~N}$ mice with spared nerve injury, ${ }^{8}$ and the hawthorn fruit is a promising remedy in the treatment of anxiety ${ }^{9}$ and has also been reported to reduce depressive-like behaviors in mice with chronic mild stress. ${ }^{7}$ Furthermore, a possible mechanism of reducing depression is increasing brainderived neurotrophic factor (BDNF) levels. ${ }^{10}$ However, the effect of Radix Puerariae and hawthorn fruit on depression associated with type 2 diabetes remains unknown. In the present study, the antidepressant effect of ethanol extract of Radix Puerariae and hawthorn fruit on a rat model with diabetes was assessed, and by using open field test (OFT), elevated plus-maze test (EPMT), locomotor activity test (LAT), and the forced swimming test (FST), it was observed that the combination markedly restored the body weight and improved the performances of rats with type 2 diabetes, indicating a possible antidepressant activity. The levels of extracellular signal-regulated protein kinases (ERKs) and BDNF in the prefrontal cortex of rats were also assessed in order to obtain further insight into the mechanism of the antidepressant action of the combination of Radix Puerariae and hawthorn fruit (CRPHF).

\section{Materials and methods Drugs and reagents}

The extracts of Radix Puerariae and hawthorn fruit were provided by the Shanghai Chinese Traditional Medical University. These extracts were separated by using the following process: $1 \mathrm{~kg}$ of each extract was reflux-extracted twice with $60 \%$ alcohol; the amount of solvent used was $5 \mathrm{~L}$, and the extraction time was 90 min for each extraction. ${ }^{11}$ The extract was evaporated at reduced pressure until the volume became $590 \mathrm{~mL}$. The Radix Puerariae and hawthorn fruit solutions were mixed at a volume ratio of $1: 1$ and used for treating the rats in this study.

\section{Animals and experimental design}

A total of 24 male Sprague Dawley rats, aged 6-8 weeks (200-250 g), were obtained from the Experimental Animal Center of Zhejiang Province (Zhejiang, People's Republic of China). Rats were given free access to food and water throughout the experiment. All the animal-handling procedures were in accordance with the Regulations for the Administration of Affairs Concerning Experimental Animals. The study was approved by the Ethics Committee of Ningbo University. All the surgical procedures were performed under anesthesia, and all efforts were made to minimize suffering.

The rats were randomly assigned to four groups ( $n=6$ per group) as follows: 1) normal control (N) group, consisting of rats fed with the regular diet; 2) normal-given-CRPHF (NC) group, consisting of rats fed with the regular diet and treated with CRPHF; 3) diabetic control (D) group, consisting of rats fed with the high-fat diet; and 4) diabetic-givenCRPHF (DC) group, consisting of rats fed with the high-fat diet and treated with CRPHF. The entire experimental period was 9 weeks, and the rats were fed a regular or high-fat diet throughout this time period according to their group assignment. Streptozotocin (STZ) was injected at the end of the 4th week. Rats in the D and DC groups were given an intraperitoneal injection of $25 \mathrm{mg} / \mathrm{kg}$ STZ (Sigma, St Louis, MO, USA) dissolved in a citrate buffer with a $\mathrm{pH}$ of 4.5. Rats in the $\mathrm{N}$ and $\mathrm{NC}$ groups were injected with the same volume of the citrate buffer. After STZ injection, random blood glucose (RBG) levels were measured daily, and an additional equivalent dose of STZ was given to the rats whose RBG was $<11.1 \mathrm{mmol} / \mathrm{L}$ on the $3 \mathrm{rd}$ day. On the 7 th day after the initial STZ injection, RBG of all the rats in D and DC groups was found to be $>11.1 \mathrm{mmol} / \mathrm{L}$, indicating the successful induction of diabetes. In the last 4 weeks, the rats were treated with CRPHF or vehicle. For the treatment, the rats were administered CRPHF ( $5 \mathrm{~mL} / \mathrm{kg} / \mathrm{d}$, NC and DC groups), or an equivalent volume of normal saline ( $\mathrm{N}$ and $\mathrm{D}$ groups), daily for 4 weeks through an intragastric feeding tube.

\section{Tissue preparation}

After the treatment was completed, all the rats were euthanized by intraperitoneal injection of a lethal dose of pentobarbital. The abdominal cavity was opened, and blood samples 
were collected by direct puncture of the descending aorta into ethylenediaminetetraacetic acid anticoagulant tubes and then centrifuged at $3,000 \mathrm{rpm} / \mathrm{min}$ for $15 \mathrm{~min}$ to collect the serum. Sera were stored frozen until use. The skull was opened, and the brain was immediately frozen in liquid nitrogen for further analysis or fixed in $4 \%$ paraformaldehyde at $4^{\circ} \mathrm{C}$ for $24 \mathrm{~h}$.

\section{Measurement of body weight and RBG}

Body weights were recorded at 7 days after the injection of STZ and again at the end of the experiment. Blood was collected from the tail vein, and RBG was measured with a blood glucose meter once every 4 days in the last 4 weeks of the experiment (Johnson \& Johnson, New Brunswick, NJ, USA). Fasting blood glucose measurement and oral glucose tolerance test (OGTT) were performed on overnight-fasted rats in order to evaluate glucose tolerance at the end of the study. Rats were fasted for $12 \mathrm{~h}$ and then given a glucose solution ( $2 \mathrm{~g} / \mathrm{kg}$ body weight), and blood samples were collected before and at 30,60, and 120 min after the glucose solution was given.

\section{Determination of blood lipid parameters}

Blood lipid parameters were measured at the end of the study. Sera prepared as described earlier were used for the detection of high-density lipoprotein cholesterol (HDL-C), lowdensity lipoprotein cholesterol, total cholesterol (TC), and triglyceride (TG) by using a MODULAR P800 Automated Biochemist Analyzer (Roche, Basel, Switzerland).

\section{OFT}

The OFT was conducted in order to measure spontaneous activity in rodents in a novel environment. ${ }^{12}$ Briefly, the rats were placed individually in a white Plexiglas box $(100 \times 100 \times 40 \mathrm{~cm})$ with the bottom divided into four identical squares on the floor of the arena. The test room was dimly illuminated. A single rat was placed in the center of the cage. Line crossing (four paws placed into a new square) and rearing (with both front paws raised from the floor) were recorded over 5 min by using the EthoVision video tracking system (Noldus, Leesburg, VA, USA). All behaviors were recorded by using a video camera located $150 \mathrm{~cm}$ above the arena. After each trial, the arena was cleaned with $75 \%$ alcohol.

\section{EPMT}

EPMT is a widely used behavioral assay for anxiety responses to evaluate risk-taking behavior. ${ }^{12}$ The elevated plus-maze apparatus has a plus sign-like shape and consists of two "open" (no walls, $50 \times 10 \mathrm{~cm}$ ) and two "closed" $(50 \times 10 \times 40 \mathrm{~cm}) \mathrm{arms}$, arranged perpendicularly and elevated $70 \mathrm{~cm}$ above the floor. Each animal was placed at the center of the device, facing an open arm, and then were allowed to freely explore the maze for $5 \mathrm{~min}$, and all the testing sessions were conducted between 5:00 pm and 10:00 pm in a sound-attenuated room. After each trial, the test maze was wiped with $75 \%$ ethanol. The number of entries into the open and closed arms, and the time spent in the open and closed arms were recorded. The percentage of time spent in the open arms and the percentage of open arm entries were calculated on the basis of the total time and the total number of entries (number of entries in open + closed arms) and were used as anxiety and locomotor parameters.

\section{LAT}

The LAT was used to evaluate depressive-like and anxietylike behaviors ${ }^{13}$ as a complement to OFT. Each rat was placed in a black box $(100 \times 100 \times 40 \mathrm{~cm})$ without cover, which was enclosed in a soundproof box. The test room was kept relatively quiet. Horizontal distance was measured by the sequential breaking of infrared beams. Movement time was incremented when a rat was active for $0.1 \mathrm{~s}$. The average speed of movement was obtained by dividing the total horizontal distance moved by the total time in motion for each rat in the $60 \mathrm{~min}$ testing period, and locomotor activity (distance moved in $\mathrm{cm}$ ) was used to evaluate depressive-like and anxiety-like behaviors. After each trial, the apparatus was cleaned with $75 \%$ ethanol.

\section{FST}

The FST was conducted to assess behavioral despair in a sound-attenuated room, which was dimly illuminated. ${ }^{14}$ Rats were placed individually in a clear plastic cylinder (height $=40 \mathrm{~cm}$; diameter $=30 \mathrm{~cm}$ ) filled with water to $21.5 \pm 1.5 \mathrm{~cm}$ high at $24^{\circ} \mathrm{C} \pm 0.5^{\circ} \mathrm{C}$ for $5 \mathrm{~min}$. The rats were allowed to swim in the cylinder under the condition that escape was not possible. The duration of immobility, which is defined as the lack of motion of the whole body except for small movement necessary to keep the head above the water, was recorded, and the extent of despair symptoms was evaluated in the rats. After each trial, the cylinder was cleaned and filled with freshwater.

\section{Protein extraction and Western blot analysis}

Proteins were extracted from rat brain tissues that were frozen in liquid nitrogen; $10 \mathrm{mg}$ of brain tissues was homogenized in a lysis buffer containing $200 \mu \mathrm{L}$ RIPA (Solarbio Science \& Technology Co., Ltd, Beijing, People's Republic of China) 
and $1 \mathrm{mM}$ aprotinin, and the lysate was centrifuged at $12,000 \times \mathrm{rpm}$ for $5 \mathrm{~min}$. The protein concentration of the supernatant was quantified by using a Bicinchoninic Acid Protein Assay kit (Beyotime Institute of Biotechnology, Shanghai, People's Republic of China). For Western blot analysis, ${ }^{15}$ the protein samples were mixed in a ratio of $4: 1$ with a sample buffer (Beyotime Institute of Biotechnology) and boiled for $5 \mathrm{~min}$. Samples (containing $80 \mu \mathrm{g}$ of proteins in total) were resolved by $12 \%$ sodium dodecyl sulfate-polyacrylamide gel electrophoresis. Separated proteins were electrophoretically transferred to polyvinylidene fluoride membranes (Immobilon-P; Millipore Corp., Billerica, MA, USA) in a buffer containing $38.67 \mathrm{mM}$ glycine, $47.9 \mathrm{mM}$ Tris, and 20\% methanol. Nonspecific binding was blocked by incubation of the membranes with 10\% nonfat milk in phosphate-buffered solution (PBS) containing 0.1\% Tween-20 (PBST) for $1 \mathrm{~h}$ at room temperature. Then, the membranes were incubated with the following primary antibodies diluted in $1 \%$ milk in PBST overnight at $4^{\circ} \mathrm{C}$ : primary antibodies for BDNF (Santa Cruz Biotechnology, Santa Cruz, CA, USA; 1:200), p-ERK (Cell Signaling Technology, Danvers, MA, USA; 1:500), ERK (Cell Signaling Technology, 1:1,000), and $\beta$-actin (Cell Signaling Technology, 1:5,000). Anti-rabbit and anti-mouse secondary antibodies were used at a ratio of 1:5,000. The membranes were washed with PBST, and then, they were incubated with horseradish peroxidase-conjugated secondary antibodies for $1 \mathrm{~h}$ and visualized by chemiluminescence (Tanon-5,200 gel imager, Shanghai, People's Republic of China). The relative expression of proteins was quantified by densitometry scanning of the blots and calculated by using the ImageJ $2 \mathrm{X}$ software (National Institutes of Health, Bethesda, MD, USA).

\section{Statistics}

Statistical analysis was conducted by using GraphPad Prism ${ }^{\circledR}$ version 5.02 (GraphPad Software, La Jolla, CA, USA), and the accepted significance level for all the tests was $P<0.05$. All the data were expressed as mean \pm standard deviation; $t$-test of independent samples was used to compare the differences between the two groups, whereas one-way analysis of variance was used to compare the differences among the three or more groups.

\section{Results \\ RBG and OGTT}

A total of 24 rats were used in the in vivo experiment, and they all survived until the end of the experiment. As shown in Figure 1A, between- and within-group analyses were performed to identify the differences in RBG across the various groups and time points, respectively. The rats in $\mathrm{N}$ group exhibited stable RBG levels $(5.53-6.23 \mathrm{mmol} / \mathrm{L})$ throughout the treatment period, whereas the RBG of rats in D group showed an increasing trend and the $\mathrm{RBG}$ of $\mathrm{NC}$ and $\mathrm{DC}$ groups given the CRPHF showed decreasing trend. The percent reduction in RBG was 18.7 and 54.9 on Day 28 (the day after the last CRPHF treatment, $4.82 \pm 0.50 \mathrm{mmol} / \mathrm{L}, 8.45 \pm 2.17 \mathrm{mmol} / \mathrm{L}$ ) compared with Day 1 (the first day of CRPHF treatment, $5.93 \pm 0.86 \mathrm{mmol} / \mathrm{L}, 18.75 \pm 5.59 \mathrm{mmol} / \mathrm{L})$ in $\mathrm{NC}$ and $\mathrm{DC}$ groups, respectively. Between-group analysis showed that the RBG level of rats in the DC group was significantly lower than that of rats in the D group on Day $28(P<0.01)$. OGTT was conducted to determine whether CRPHF improved glucose homeostasis in these animals. As shown in Figure 1B, all the groups showed significant increases $(P<0.001)$ in RBG $1 \mathrm{~h}$
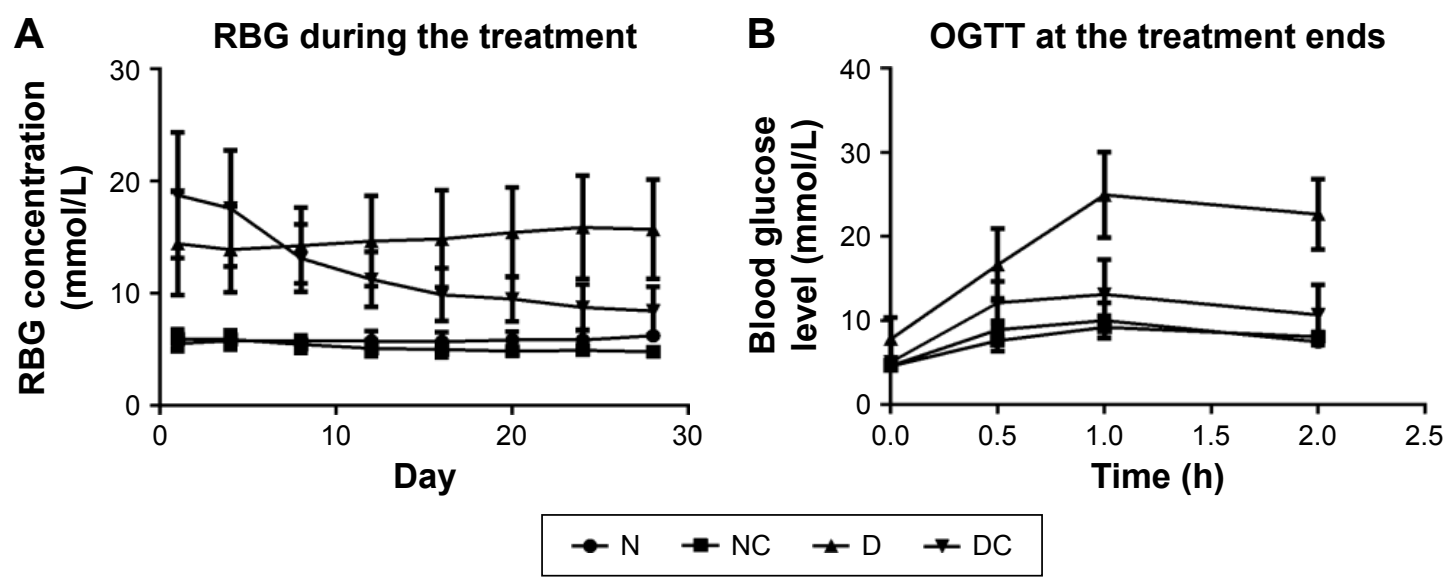

Figure I CRPHF reduced random blood glucose and improved glucose tolerance in diabetic rats.

Notes: Day I, the first of the treatment period. Diabetic rats given CRPHF showed a downward trend in random blood glucose levels during the CRPHF treatment period (A) and an improved glucose tolerance as measured by OGTT at the end of the treatment (B) compared with diabetic control rats.

Abbreviations: CRPHF, combination of Radix Puerariae and hawthorn fruit; D, diabetic control; DC, diabetic-given-CRPHF; N, normal control; NC, normal-given-CRPHF; OGTT, oral glucose tolerance test; RBG, random blood glucose. 
following oral glucose challenge caused by the oral glucose. It was found that RBG showed a greater increase in the D group than in the DC group from $0.5 \mathrm{~h}$ to $1 \mathrm{~h}$, indicating a postponed RBG peak. Moreover, rats in the DC group had lower RBG compared with the D group at $2 \mathrm{~h}$ after the glucose challenge. Interestingly, the glucose levels in the DC group rats remained $52.8 \%$ lower than that of the D group at $2 \mathrm{~h}$ after the glucose challenge, indicating that rats treated with CRPHF had improved glucose tolerance.

\section{CRPHF improved lipid metabolism in diabetic rats}

CRPHF reduced TG $(1.79 \pm 0.27 \mathrm{mmol} / \mathrm{L}, 2.65 \pm 0.44 \mathrm{mmol} / \mathrm{L}$, $P<0.01)$ and TC $(1.74 \pm 0.08 \mathrm{mmol} / \mathrm{L}, 2.23 \pm 0.22 \mathrm{mmol} / \mathrm{L}$, $P<0.001$ ) in diabetic rats (Figure 2). Similarly, the NC group showed significantly decreased TG $(1.11 \pm 0.08 \mathrm{mmol} / \mathrm{L}$, $1.52 \pm 0.22 \mathrm{mmol} / \mathrm{L}, P<0.05)$ and TC $(1.24 \pm 0.06 \mathrm{mmol} / \mathrm{L}$, $1.49 \pm 0.02 \mathrm{mmol} / \mathrm{L}, P<0.001)$ and significantly increased HDL-C $(1.20 \pm 0.18 \mathrm{mmol} / \mathrm{L}, 0.92 \pm 0.17 \mathrm{mmol} / \mathrm{L}, P<0.05)$ compared with the $\mathrm{N}$ group. These data indicate that $\mathrm{CRPHF}$ effectively improved lipid metabolism in type 2 diabetic rats and lowered blood lipid in normal rats to a certain extent.

\section{CRPHF restored the body weight of diabetic rats}

There was a gradual increase in the body weights of the rats during the first 4 weeks of feeding period. As shown in Figure 3A, on the first day of treatment, the mean body weights of the high-fat diet groups $(430 \pm 6.50 \mathrm{~g})$ were significantly higher than those in the corresponding normal diet groups (485 $\pm 20.56 \mathrm{~g}, P<0.05)$. At the completion of the study (Figure 3B), the mean body weights in the DC group $(494 \pm 44.44 \mathrm{~g})$ were significantly higher than those in the D group (446 $\pm 22.10 \mathrm{~g} ; P<0.05)$, and similarly, the mean body weights in the $\mathrm{NC}$ group were significantly higher than those in the $\mathrm{N}$ group $(P<0.001)$. The body weights of rats in the $\mathrm{D}$ group were less than that of the $\mathrm{N}$ group, resembling the weight loss seen in diabetic patients. These findings suggested that the CRPHF could significantly restore the reduction in body weight caused by STZ.
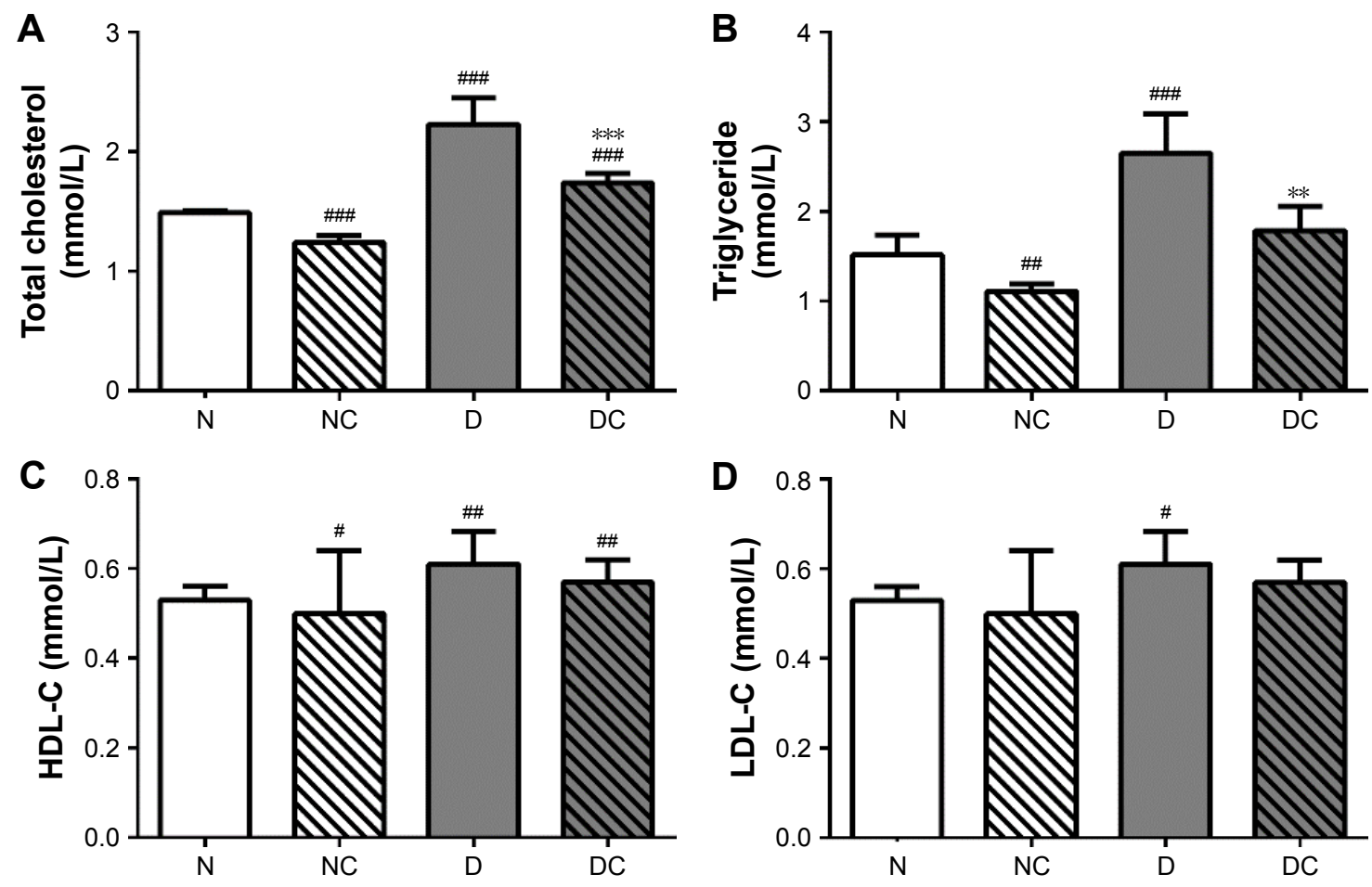

$\begin{array}{llll}\mathrm{N} & \mathrm{V} C & \mathrm{D} & \mathbf{\nabla} \mathrm{DC}\end{array}$

Figure 2 CRPHF reduced blood lipid in diabetic rats.

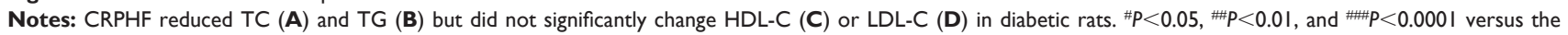
$\mathrm{N}$ group and $* * \mathrm{P}<0.0 \mathrm{I}$ and $* * * \mathrm{P}<0.000 \mathrm{I}$ versus the $\mathrm{D}$ group ( $\mathrm{n}=6$ per group).

Abbreviations: CRPHF, combination of Radix Puerariae and hawthorn fruit; D, diabetic control; DC, diabetic-given-CRPHF; HDL-C, high-density lipoprotein cholesterol; LDL-C, low-density lipoprotein cholesterol; N, normal control; NC, normal-given-CRPHF; TC, total cholesterol; TG, triglyceride. 

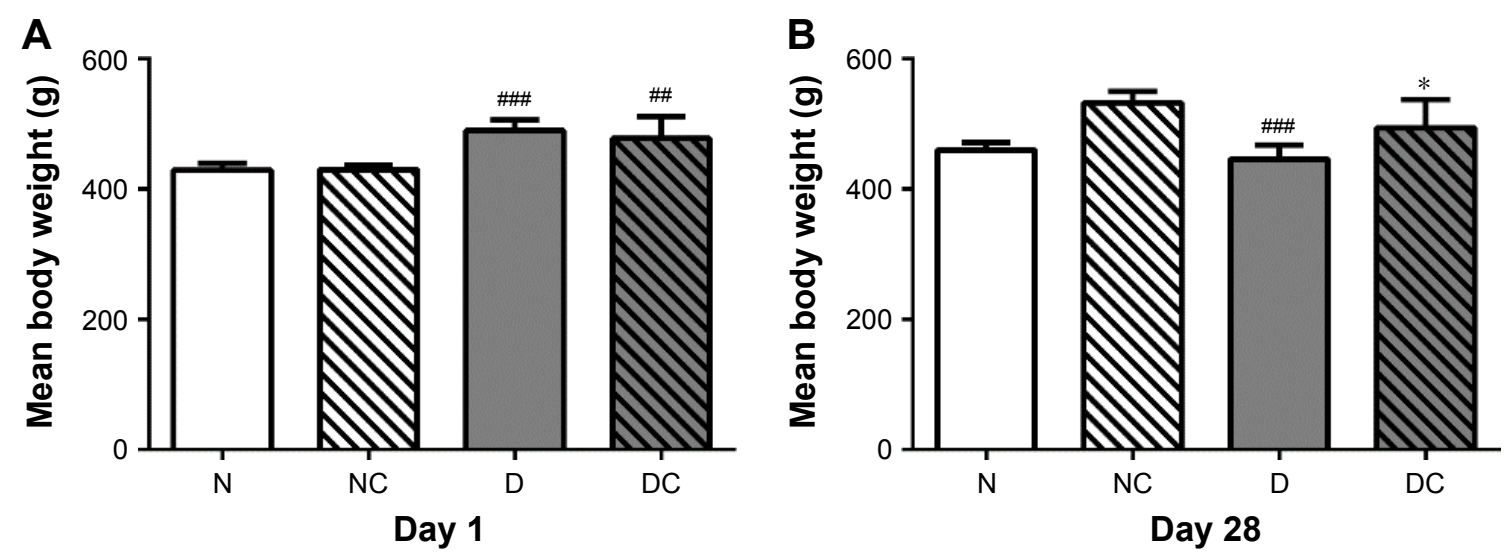

$\square N \boldsymbol{\nabla N C} \square D \boldsymbol{\nabla D C}$

Figure $3 \mathrm{CRPHF}$ restored the body weights in diabetic rats.

Notes: Left panel $(\mathbf{A})$ shows the body weights immediately after the injection of STZ. Right panel (B) illustrates that CRPHF reversed the body weight reduction in diabetic rats. ${ }^{\#} P<0.01$ and ${ }^{\#} P<0.000$ I versus the $N$ group and $* P<0.05$ versus the $D$ group ( $n=6$ per group).

Abbreviations: CRPHF, combination of Radix Puerariae and hawthorn fruit; D, diabetic control; DC, diabetic-given-CRPHF; N, normal control; NC, normal-given-CRPHF; STZ, streptozotocin.

\section{CRPHF reduced depressive-like behaviors in diabetic rats}

Depressive-like behaviors were assessed by using four different tests (Figure 4). High-fat diet and STZ injection induced a marked decrease in the frequency of line crossing and rearing during OFT (Figure 4A and B; $P<0.001$ for both), a significant decrease in the total time and entries in the open arms in the EPMT (Figure 4C and D; $P<0.05$, $P<0.001$, respectively) and a pronounced decrease in LAT (Figure 4E; $P<0.001$ ) compared with the N group. CRPHF significantly reversed the decreases in the measured parameters in OFT, EPMT, and LAT ( $P<0.001$ for all) induced by DM. For FST (Figure 4F), immobility time was prolonged in the $\mathrm{D}$ group versus the $\mathrm{N}$ group $(P<0.001)$. CRPHF treatment significantly alleviated the STZ-induced increase in immobility time $(P<0.001)$.

\section{CRPHF increased the protein level of phosphorylated extracellular signal- regulated kinase ( $p-E R K)$ and $B D N F$ in the brain of diabetic rats}

$\mathrm{BDNF}$ is a neurotrophin that is critical for the control of mood, emotion, learning, and cognition, and a number of animal and human studies have suggested the role of BDNF in the pathogenesis of recurrent mood disorders. ${ }^{16}$ In addition, serum BDNF levels are abnormally low in patients with depressive disorder. ${ }^{17}$ Moreover, a meta-analysis suggests a potential role of BDNF as a marker of treatment response after treatment in patients with mood disorders, ${ }^{18}$ and another study showed that p-ERK levels decreased in depression rats. ${ }^{19}$ Therefore, the BDNF and p-ERK expressions were measured to ensure the success of depression rat models and to evaluate the therapeutic effect of CRPHF at the molecular level. As shown in Figure 5, the protein levels of $\mathrm{p}$-ERK and BDNF were significantly higher $(P<0.05$ and $P<0.001$, respectively) in the brain of the rats in DC group than in the rats in the D group. Moreover, the protein levels of p-ERK and BDNF were lower in rats of the D group than in rats of the $\mathrm{N}$ group $(P<0.05$ and $P<0.01$, respectively).

\section{Discussion}

Chronic depression is frequent among diabetic patients, aggravates DM-induced complications, and increases mortality. ${ }^{20,21}$ The pathophysiological connections between DM and depression have not yet been widely studied. The present study showed that DM induced behavioral changes that can be measured by OFT, EPMT, LAT, and FST, whereas CRPHF reversed these behavioral changes. It further showed that the p-ERK-BDNF pathway might be associated with the comorbidity of these diseases.

Appropriate diabetic animal models play an important role in illustrating the underlying mechanisms of $\mathrm{DM}^{22}$ and the associated depression. The high-fat diet- and STZ-induced type 2 diabetic rat model is well accepted as this model is closely analogous to the clinical situation in humans, ${ }^{23}$ and the hyperglycemia state (Figure 1) and hyperlipidemia state (Figure 2) also conform to the transitions of depression in the progression of type 2 diabetes. To the best of the authors' knowledge, the present study is the first to demonstrate that the high-fat diet and STZ-induced type 2 diabetic rat model exhibits depressive-like behaviors. 
A

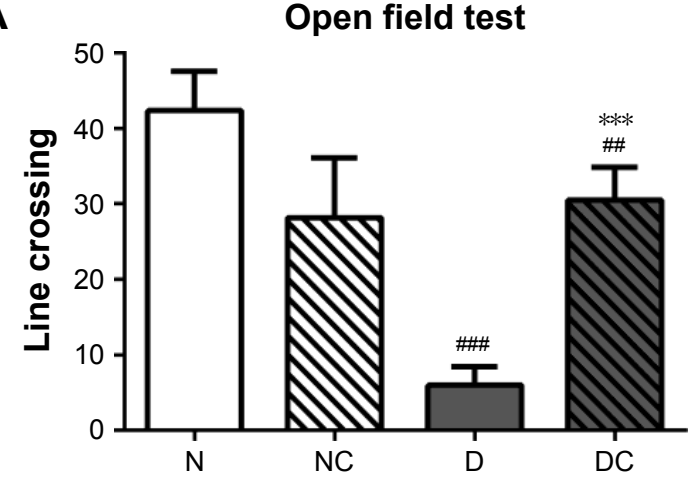

C

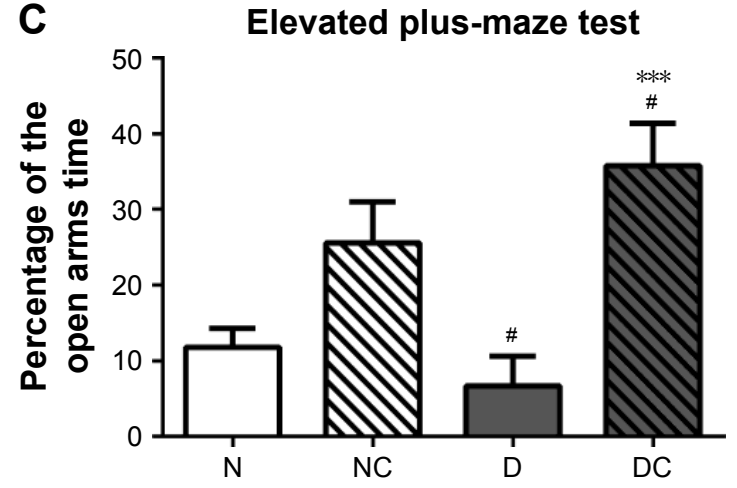

E

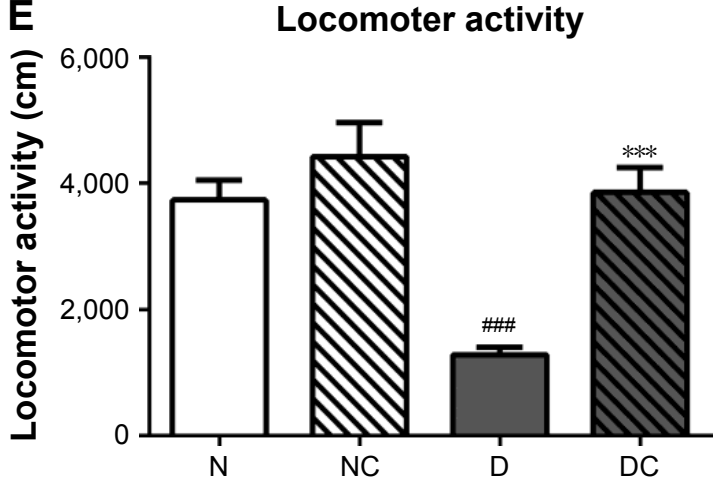

B

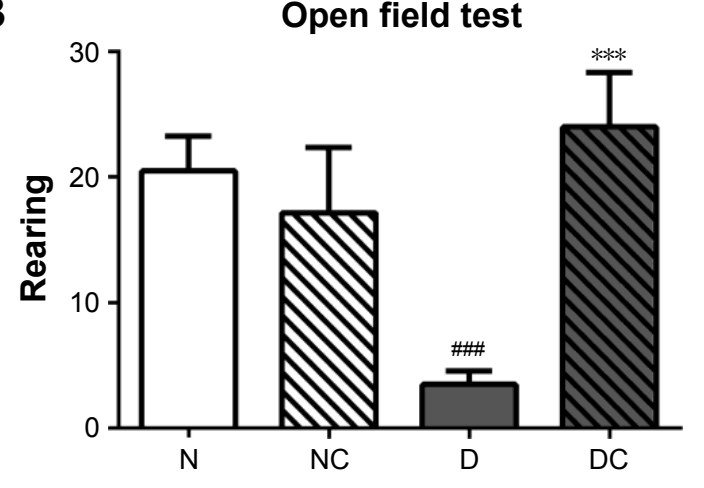

D

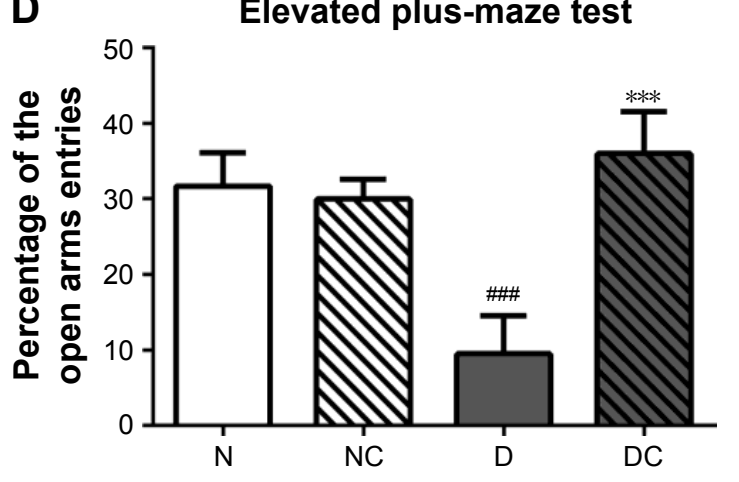

F

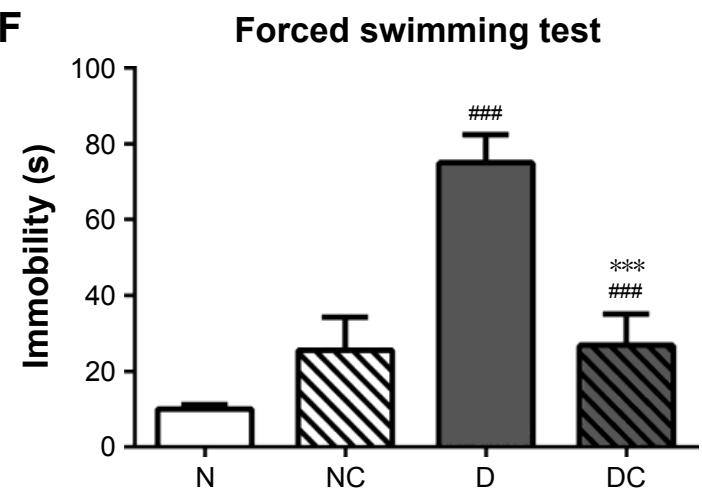

Figure 4 CRPHF reduced depressive-like behaviors in diabetic rats.

Notes: (A) and (B) CRPHF increased the frequency of line crossing and rearing in the open field test; (C) and (D) CRPHF increased the total time and the number of entries in the open arms in the elevated plus-maze test; (E) CRPHF increased the locomotor activity of diabetic rats; (F) CRPHF decreased the immobility time of rats in the forced swimming test. ${ }^{\#}<0.05,{ }^{\# P}<0.01$, and ${ }^{\#} P<0.000$ I versus the $N$ group and $* * * P<0.000$ I versus the $D$ group (n=6 per group).

Abbreviations: CRPHF, combination of Radix Puerariae and hawthorn fruit; D, diabetic control; DC, diabetic-given-CRPHF; N, normal control; NC, normal-givenCRPHF.

Previous studies have shown the therapeutic effects of Traditional Chinese Medicine in treating patients with depression or DM. The present study showed the dual function of a novel herbal treatment to reduce RBG and depressive-like behaviors. Treatment with a dose of $2 \mathrm{~g} / \mathrm{kg} / \mathrm{d}$ CRPHF for 4 weeks reversed DM-induced depressive-like moving pattern in type 2 diabetic rats, suggesting that long-term administration of CRPHF reduces depressive-like behaviors.

Further research into the mechanism of the observed effects showed that treatments with CRPHF have increased p-ERK and BDNF levels in the prefrontal cortex. p-ERK is implicated in rapid antidepressant effects, ${ }^{24}$ thus, it is possible that p-ERK contributes to the antidepressant effects of CRPHF. BDNF is a critical regulator of the formation, regulation, and integrity of neurons in brain circuits that regulate emotion. ${ }^{25}$ The neurotrophic hypothesis suggests that reduced activity of BDNF causes a depressive state. ${ }^{26}$ Decreased central ${ }^{27}$ and peripheral levels of $\mathrm{BDNF}^{28}$ have been reported in patients with major depression disorder. Associations between $B D N F$ gene and major depression disorder have been reported in several human genetic studies. ${ }^{29,30}$ Some other studies have found reduced mRNA levels of BDNF in the prefrontal cortex and hippocampus of suicide subjects, compared with the control subjects. ${ }^{31}$ 

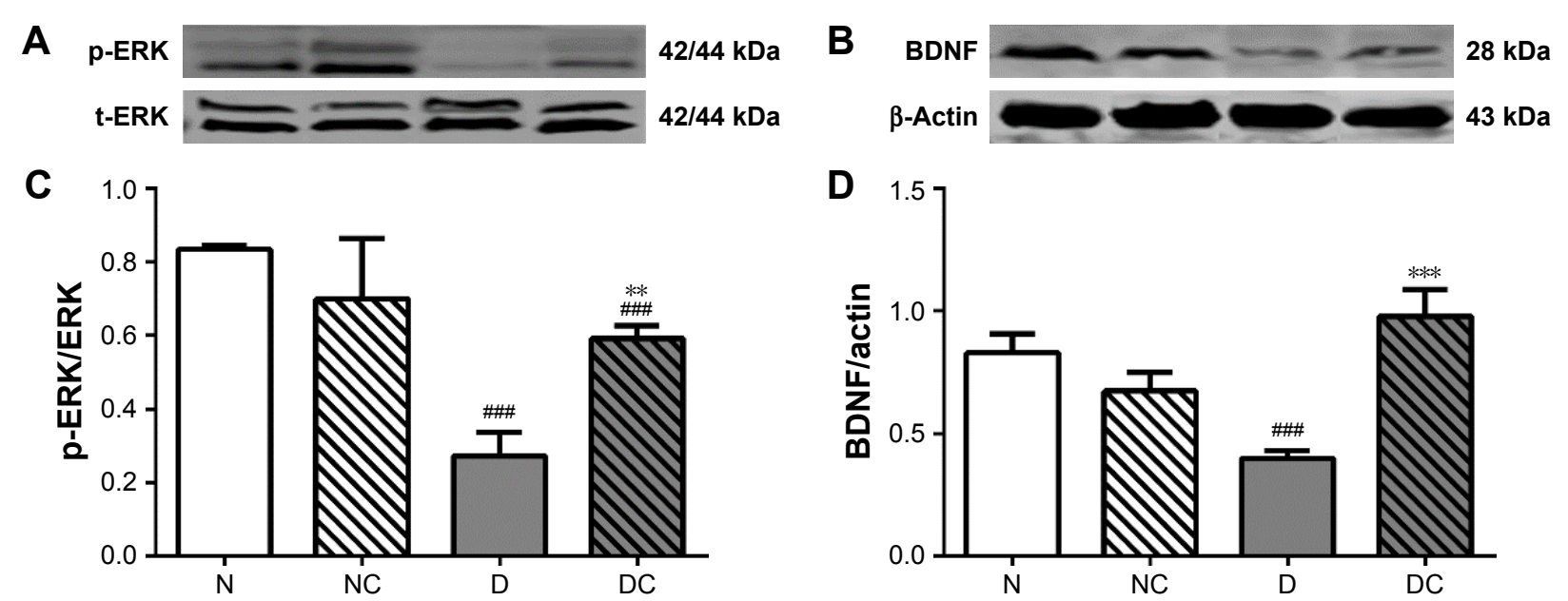

Figure 5 CRPHF increased the protein levels of p-ERK and BDNF in the brain of diabetic rats.

Notes: Top panels $(\mathbf{A})$ and $(\mathbf{B})$ are representative images of immunoblots of $42 / 44 \mathrm{kDa}$-ERK and $28 \mathrm{kDa}$ BDNF; lower panels (C) and (D) show the protein level of $\mathrm{P}$-ERK and $B D N F$ in the brain of rats. All data are shown as mean \pm standard deviation; $n=6$ per group. $P<0.000 \mathrm{I}$ versus the $\mathrm{N}$ group and $* * P<0.0 \mathrm{I}$ and $* * * P<0.000 \mathrm{I}$ versus the D group.

Abbreviations: BDNF, brain-derived neurotrophic factor; CRPHF, combination of Radix Puerariae and hawthorn fruit; D, diabetic control; DC, diabetic-given-CRPHF; $\mathrm{N}$, normal control; NC, normal-given-CRPHF; p-ERK, phosphorylated extracellular signal-regulated protein kinase.

Consistent with these findings, the present study showed that BDNF is decreased when rats are exposed to a high-fat diet for 4 weeks followed by an STZ injection, and CRPHF treatment increases BDNF levels in the prefrontal cortex in these diabetic rats.

\section{Conclusion}

In conclusion, a depression-like rat model was developed based on a high-fat diet and STZ injection. The results of the present study suggest that CRPHF is a putative antidepressant for patients with diabetes, and it possibly acts through the p-ERK-BDNF pathway. Further research is required to investigate the optimum dose of CRPHF and exact mechanisms underlying these effects.

\section{Acknowledgments}

This work was supported by the Ningbo Science and Technology Innovation Team Program (2014B82002); the Natural Science Foundation of Ningbo (2015A610176); the Natural Science Foundation of Zhejiang province (Q16H100001); the National Natural Science Foundation of China (281370165, 81501421); the Fang Runhua Fund of Hong Kong; and KC Wong Magna Fund in Ningbo University.

\section{Disclosure}

The authors report no conflicts of interest in this work.

\section{References}

1. Musselman DL, Betan E, Larsen H, Phillips LS. Relationship of depression to diabetes types 1 and 2: epidemiology, biology, and treatment. Biol Psychiatry. 2003;54(3):317-329.
2. Bogner HR, Morales KH, de Vries HF, Cappola AR. Integrated management of type 2 diabetes mellitus and depression treatment to improve medication adherence: a randomized controlled trial. Ann Fam Med. 2012;10(1):15-22.

3. Anderson RJ, Freedland KE, Clouse RE, Lustman PJ. The prevalence of comorbid depression in adults with diabetes: a meta-analysis. Diabetes Care. 2001;24(6):1069-1078.

4. Habtewold TD, Alemu SM, Haile YG. Sociodemographic, clinical, and psychosocial factors associated with depression among type 2 diabetic outpatients in Black Lion General Specialized Hospital, Addis Ababa, Ethiopia: a cross-sectional study. BMC Psychiatry. 2016;16(1):103.

5. Ishizawa K, Babazono T, Horiba Y, et al. The relationship between depressive symptoms and diabetic complications in elderly patients with diabetes: analysis using the Diabetes Study from the Center of Tokyo Women's Medical University (DIACET). $J$ Diabetes Complications. 2016;30(4):597-602.

6. Yan B, Wang DY, Xing DM, et al. The antidepressant effect of ethanol extract of radix puerariae in mice exposed to cerebral ischemia reperfusion. Pharmacol Biochem Behav. 2004;78(2):319-325.

7. Doron R, Lotan D, Einat N, et al. A novel herbal treatment reduces depressive-like behaviors and increases BDNF levels in the brain of stressed mice. Life Sci. 2014;94(2):151-157.

8. Zhao J, Luo D, Liang Z, Lao L, Rong J. Plant natural product puerarin ameliorates depressive behaviors and chronic pain in mice with spared nerve injury (SNI). Mol Neurobiol. Epub 2016 Mar 24.

9. Haselbeck A. Epoetins: differences and their relevance to immunogenicity. Curr Med Res Opin. 2003;19(5):430-432.

10. Li Q, Zhao HF, Zhang ZF, et al. Long-term administration of green tea catechins prevents age-related spatial learning and memory decline in C57BL/6 J mice by regulating hippocampal cyclic amp-response element binding protein signaling cascade. Neuroscience. 2009; 159(4):1208-1215.

11. Fu X, Song B, Tian GW, Li JL. The effects of the water-extraction of Astragali Radix and Lycopi herba on the Pathway of TGF-smads-UPP in a rat model of diabetic nephropathy. Pharmacogn Mag. 2014;10(40): 491-496.

12. Meirsman AC, Robé A, de Kerchove d'Exaerde A, Kieffer BL. GPR88 in A2AR neurons enhances anxiety-like behaviors. eNeuro. 2016; 3(4):pii.

13. Burke NN, Coppinger J, Deaver DR, Roche M, Finn DP, Kelly J. Sex differences and similarities in depressive- and anxiety-like behaviour in the Wistar-Kyoto rat. Physiol Behav. 2016;167:28-34. 
14. Nelson LH, Lenz KM. Microglia depletion in early life programs persistent changes in social, mood-related, and locomotor behavior in male and female rats. Behav Brain Res. 2017;316:279-293.

15. Fujita N, Shimotake N, Ohki I, et al. Mechanism of transcriptional regulation by methyl-CpG binding protein MBD1. Molecular and Cellular Biology. 2000;20(14):5107-5118.

16. Post RM. Role of BDNF in bipolar and unipolar disorder: clinical and theoretical implications. J Psychiatr Res. 2007;41(12):979-990.

17. Sen S, Duman R, Sanacora G. Serum brain-derived neurotrophic factor, depression, and antidepressant medications: meta-analyses and implications. Biol Psychiatry. 2008;64(6):527-532.

18. Rocha RB, Dondossola ER, Grande AJ, et al. Increased BDNF levels after electroconvulsive therapy in patients with major depressive disorder: a meta-analysis study. J Psychiatr Res. 2016;83:47-53.

19. Wang X, Xie Y, Zhang T, et al. Resveratrol reverses chronic restraint stress-induced depression-like behaviour: involvement of BDNF level, ERK phosphorylation and expression of Bcl-2 and Bax in rats. Brain Res Bull. 2016;125:134-143.

20. Katon W, Fan MY, Unützer J, Taylor J, Pincus H, Schoenbaum M. Depression and diabetes: a potentially lethal combination. J Gen Intern Med. 2008;23(10):1571-1575.

21. Zelena D, Barna I, Mlynarik M, Gupta OP, Jezova D, Makara GB. Stress symptoms induced by repeated morphine withdrawal in comparison to other chronic stress models in mice. Neuroendocrinology. 2005;81(3): 205-215.

22. Srinivasan K, Viswanad B, Asrat L, Kaul CL, Ramarao P. Combination of high-fat diet-fed and low-dose streptozotocin-treated rat: a model for type 2 diabetes and pharmacological screening. Pharmacol Res. 2005;52(4): 313-320.

23. Skovso S. Modeling type 2 diabetes in rats using high fat diet and streptozotocin. J Diabetes Investig. 2014;5(4):349-358.
24. Xue W, Zhou X, Yi N, et al. Yueju pill rapidly induces antidepressantlike effects and acutely enhances BDNF expression in mouse brain. Evid Based Complement Alternat Med. 2013;2013:184367.

25. Duman RS, Monteggia LM. A neurotrophic model for stress-related mood disorders. Biol Psychiatry. 2006;59(12):1116-1127.

26. Chourbaji S, Brandwein C, Gass P. Altering BDNF expression by genetics and/or environment: impact for emotional and depression-like behaviour in laboratory mice. Neurosci Biobehav Rev. 2011;35(3): 599-611.

27. Karege F, Bondolfi G, Gervasoni N, Schwald M, Aubry JM, Bertschy G. Low brain-derived neurotrophic factor (BDNF) levels in serum of depressed patients probably results from lowered platelet BDNF release unrelated to platelet reactivity. Biol Psychiatry. 2005; 57(9):1068-1072.

28. Molendijk ML, Bus BA, Spinhoven P, et al. Serum levels of brain-derived neurotrophic factor in major depressive disorder: state-trait issues, clinical features and pharmacological treatment. Mol Psychiatry. 2011; 16(11):1088-1095.

29. Licinio J, Dong C, Wong ML. Novel sequence variations in the brainderived neurotrophic factor gene and association with major depression and antidepressant treatment response. Arch Gen Psychiatry. 2009; 66(5):488-497.

30. Schumacher J, Jamra RA, Becker T, et al. Evidence for a relationship between genetic variants at the brain-derived neurotrophic factor (BDNF) locus and major depression. Biol Psychiatry. 2005;58(4):307-314.

31. Dwivedi Y, Rizavi HS, Conley RR, Roberts RC, Tamminga CA, Pandey GN. Altered gene expression of brain-derived neurotrophic factor and receptor tyrosine kinase B in postmortem brain of suicide subjects. Arch Gen Psychiatry. 2003;60(8):804-815.
Neuropsychiatric Disease and Treatment

\section{Publish your work in this journal}

Neuropsychiatric Disease and Treatment is an international, peerreviewed journal of clinical therapeutics and pharmacology focusing on concise rapid reporting of clinical or pre-clinical studies on a range of neuropsychiatric and neurological disorders. This journal is indexed on PubMed Central, the 'PsycINFO' database and CAS,

\section{Dovepress}

and is the official journal of The International Neuropsychiatric Association (INA). The manuscript management system is completely online and includes a very quick and fair peer-review system, which is all easy to use. Visit http://www.dovepress.com/testimonials.php to read real quotes from published authors. 